\title{
Stable Immobilization of Mediator in Porous Carbon Material via Capping Technique for Fabricating Sensitive Enzyme-based Glucose Sensor
}

\author{
Kuniaki Nagamine, ${ }^{1,2 *}$ Ryota Izawa, ${ }^{1}$ Isao Shitanda, ${ }^{3}$ and Shizuo Tokito $1,2^{* *}$ \\ ${ }^{1}$ Graduate School of Organic Materials Science, Yamagata University, \\ 4-3-16 Jonan, Yonezawa, Yamagata 992-8510, Japan \\ ${ }^{2}$ Research Center of Organic Electronics (ROEL), Yamagata University, \\ 4-3-16 Jonan, Yonezawa, Yamagata 992-8510, Japan \\ ${ }^{3}$ Department of Pure and Applied Chemistry, Faculty of Science and Technology, Tokyo University of Science, \\ Noda, Chiba 278-8510, Japan
}

(Received August 11, 2021; accepted September 8, 2021)

Keywords: porous carbon, enzyme, biosensor, electrochemistry, capping technique

A sensitive and stable porous carbon-modified enzyme sensor based on mediated electron transfer (MET) was developed for D-glucose detection. Toluidine blue (TB) was loaded as a mediator into the pores of porous carbon particles, and the pores were capped by flavin adenine dinucleotide-dependent glucose dehydrogenase (FAD-GDH) by one-step drop-casting of TB and FAD-GDH. TB was found to be stably capped in the pores by FAD-GDH for $21 \mathrm{~d}$. The resulting sensor enabled the reproducible and sensitive detection of D-glucose with a detection limit of $6.5 \mu \mathrm{M}$. The present sensor is promising for the long-term monitoring of low concentrations of biomarkers.

\section{Introduction}

Future preventive medicine and healthcare will require noninvasive diagnostics as alternatives to conventional blood-sampling-based diagnostics. Biological fluids, such as tears, ${ }^{(1)}$ saliva, ${ }^{(2)}$ urine, ${ }^{(3)}$ and sweat, ${ }^{(4)}$ are promising candidates that have noninvasively accessible biomarkers partitioned from the blood. However, these biological fluids contain relatively low concentrations of biomarkers compared with those in blood because part of the biomarkers is partitioned from interstitial fluids through passive diffusion via transcellular or paracellular transport. ${ }^{(4)}$ For example, the concentrations of D-glucose are $1.99-22.2 \mathrm{mM}$ in interstitial fluid, $0-0.8 \mathrm{mM}$ in urine, $0.05-5 \mathrm{mM}$ in tears, $0.008-1.77 \mathrm{mM}$ in saliva, and $0.01-0.1 \mathrm{mM}$ in sweat. ${ }^{(5,6)}$ These values are much lower than blood glucose levels (2-40 mM). Therefore, highly sensitive biosensors are required to perform noninvasive analyses of these externally secreted bodily fluids.

Various types of wearable and disposable electrochemical biosensors for these biological fluids have been actively developed in recent years. ${ }^{(7)}$ Some of these devices are continuously or

\footnotetext{
*Corresponding author: e-mail: nagamine@yz.yamagata-u.ac.jp

** Corresponding author: e-mail: tokito@yz.yamagata-u.ac.jp

https://doi.org/10.18494/SAM.2021.3583
} 
temporarily attached to human body to monitor changes in the concentration of biomarkers. The active membranes for these biosensors are composed of biological materials such as enzymes, antibodies, and ionophores, as well as various artificial or biomimetic chemical compounds, which generate electrochemical signals with intensity depending on the concentration of the biomarkers. Enzymes are among the most promising of these active materials owing to their high selectivity toward analytes. Enzyme-based biosensors are divided into two categories: mediated electron transfer (MET) and direct electron transfer (DET) biosensors. So many articles about these enzyme-based biosensors and their fabrication techniques have been published, and their performances were summarized in review articles. ${ }^{(8)}$ The recent articles about enzyme-based biosensors have been focusing on a DET-type biosensors, which can utilize DET between an enzyme and an electrode, owing to its simple and academically attractive configuration despite still the limited types of enzymes (or limited applications of the DET-type biosensors). On the other hand, although MET-type enzyme sensors require an electron transfer mediator to electrically connect the enzyme and electrode, they are versatile because they can be applied to more enzymatic reactions than the DET-type sensors because many artificial mediators have been developed for the enzymes such as oxidases, acetyltransferases, and dehydrogenases, for example. In this study, we focused on a MET-type biosensor because of its versatility for various biomarkers.

In wearable applications, the mediators should not leak from the active area of the MET-type biosensor because transepidermal diffusion of the mediators into the body can result in cytotoxicity. Highly porous and conductive carbon particles have been actively applied to biofuel cells to provide stable immobilization sites for enzymes and mediators in porous structures. Trifonov and coworkers loaded mediators into the pores of mesoporous carbon nanoparticles, and the pores were capped by enzymes. ${ }^{(9,10)}$ They demonstrated the long-term stability of enzyme/mediator-loaded porous-carbon-based electrodes. Shitanda et al. used MgO-templated mesoporous carbon, where the enzymes and mediators were covalently immobilized in the pores; they demonstrated the stable operation of their biofuel cell for $15 \mathrm{~d} .{ }^{(1)}$ Another advantage of porous carbon materials is the high surface area required to load a large amount of enzyme into the pores to generate high-power biofuel cells. However, there are few reports that have examined power generation with a low concentration of substrate, in other words, the sensitivity of these MET-type porous-material-based enzyme biosensors.

This study examines the sensitivity and stability of MET-type porous-carbon-based enzyme sensors using glucose as a model analyte. The $\mathrm{MgO}$-templated porous carbon has an average pore diameter of $150 \mathrm{~nm}$ and a Brunauer-Emmett-Teller (BET) relative surface area of $300 \mathrm{~m}^{2} \mathrm{~g}^{-1}$. It is demonstrated that the sensor is sensitive to detect D-glucose. In addition, while the pores into which the mediators are loaded are much larger than the size of the enzyme [flavin adenine dinucleotide-dependent glucose dehydrogenase (FAD-GDH; EC 1,1,99,10), ${ }^{(12)}$ where the diameter of a single molecule is about $6 \mathrm{~nm}^{(13)}$, we found that the mediator-loaded pores could be stably capped by the enzyme for $21 \mathrm{~d}$. This result suggests the utility of the MET-based porous carbon-based enzyme sensor for long-term and sensitive monitoring of components included in noninvasively accessible body fluids. 


\section{Materials and Methods}

\subsection{Fabrication of GDH/TB-modified porous carbon electrode}

A gold electrode pattern (50 $\mathrm{nm}$ thickness) was prepared by thermal evaporation on a polyethylene naphthalate (PEN) film substrate (125 $\mu \mathrm{m}$ thickness) through a stencil mask. The active area $\left(0.15 \mathrm{~cm}^{2}\right)$ of the Au electrode pattern was connected to a narrow lead-line pattern (1 mm width, $3 \mathrm{~cm}$ length). Carbon-graphite ink (C2000802P2, Sun Chemical) was painted on the active area through a stencil sheet, followed by drying at $80{ }^{\circ} \mathrm{C}$ for $15 \mathrm{~min}$. The lead-line pattern was insulated by coating with a fluoropolymer ( $5 \mathrm{wt} \%$, DuPont, Teflon AF1600) layer in Fluorinert (3M, FC-43), followed by annealing for $30 \mathrm{~min}$ at $60{ }^{\circ} \mathrm{C}$ in ambient air. Thirty microliters of $1 \mathrm{mg} \mathrm{mL}^{-1}$ porous carbon powder $\left(\mathrm{CNovel}^{\circledR}\right.$, Toyo Tanso Co., Ltd.) dispersed in 1-methyl-2-pyrrolidone (FIJIFILM Wako Pure Chemical Corporation) using an ultrasonic disperser (PR-1, Thinky Co.) was drop-casted on the carbon area and dried at $80{ }^{\circ} \mathrm{C}$ for $90 \mathrm{~min}$. Ten microliters of a mixture solution composed of FAD-GDH (GLD3, EC 1,1,99,10, BBI Solutions) in $100 \mathrm{mM}$ phosphate buffer (PB, pH 7.4, Nacalai Tesque) and toluidine blue (TB, Tokyo Chemical Industry Co., Ltd.) in $100 \mathrm{mM}$ PB in a ratio of 1:1 was drop-casted on the porous-carbon-modified carbon area and dried overnight at $4{ }^{\circ} \mathrm{C}$. The resulting electrode, named the GDH/TB-modified porous carbon electrode, was washed in stirred Dulbecco's phosphate buffered saline (PBS, pH 7.4, Sigma Aldrich) for $30 \mathrm{~min}$ to remove the FAD-GDH and TB loosely bound to the porous carbon.

\subsection{Electrochemical characterization of GDH/TB-modified porous carbon electrode}

All electrochemical measurements were carried out in PBS with stirring at $300 \mathrm{rpm}$. For measurement, the GDH/TB-modified porous electrode and the $\mathrm{Ag} / \mathrm{AgCl}$ reference electrode were immersed in PBS, and their terminal lines were short-circuited with an external resistor of $50 \mathrm{k} \Omega{ }^{(14)}$ The potential difference at the external resistor was monitored at room temperature using an electrochemical analyzer (ALS model 602E. BAS Inc.) operated in open-circuit potential mode (input impedance: $1 \times 10^{12} \Omega$ ). For cyclic voltammetry tests using the same electrochemical analyzer, the GDH/TB-modified porous electrode, a Pt wire, and $\mathrm{Ag} / \mathrm{AgCl}$ were used as the working, counter, and reference electrodes, respectively.

\section{Results and Discussion}

Figure 1(a) shows SEM images of the surfaces of the flat and porous-carbon-particlemodified carbon electrodes. The assembled porous carbon particles formed a microporous surface on the electrode (the average pore size identified from the SEM image was several tens of micrometers). Figure 1(b) shows cyclic voltammograms (CVs) of $10 \mathrm{mM} \mathrm{Fe}(\mathrm{CN})_{6}{ }^{4-}$ in PBS, detected using the flat and porous-carbon-particle-modified carbon electrodes. The reversible redox reaction of $\mathrm{Fe}(\mathrm{CN})_{6}{ }^{4-}$ occurred at the flat carbon electrode with an oxidation peak current of $0.24 \mathrm{~mA}$. The theoretical peak current at room temperature, calculated using the Randles- 


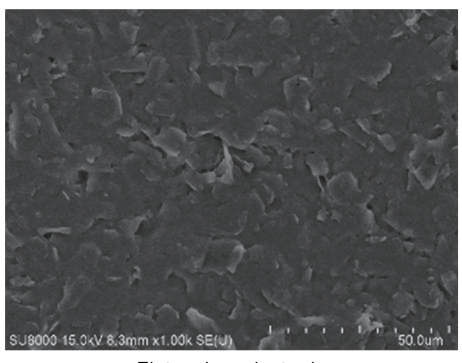

Flat carbon electrode

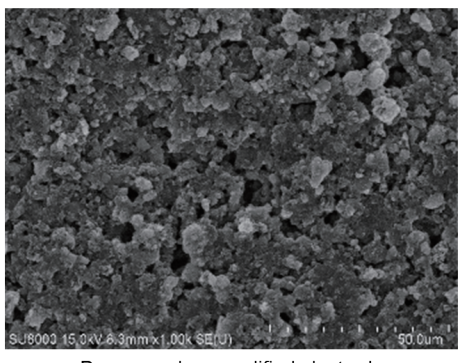

Porous carbon-modified electrode

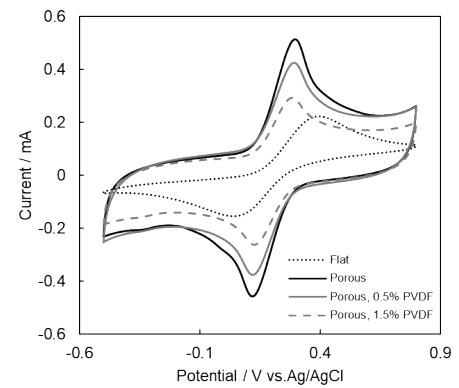

(b)

Fig. 1. (a) SEM images of surfaces of flat and porous-carbon-particle-modified carbon electrode. (b) CVs of $10 \mathrm{mM} \mathrm{Fe}(\mathrm{CN})_{6}{ }^{4-}$ in PBS, detected using flat carbon electrode and carbon electrodes modified with porous carbon particle mixture solution without and with 0.5 and $1.5 \%$ poly(vinylidene fluoride) (PVDF). Scan rate: $0.1 \mathrm{~V} \mathrm{~s}^{-1}$.

Sevcik equation, ${ }^{(15)}$ was $0.32 \mathrm{~mA}$ [the diffusion coefficient was $6.5 \times 10^{-6} \mathrm{~cm}^{2} \mathrm{~s}^{-1}{ }^{(16)}$ ]. This detected value is relatively small compared with the theoretical value, possibly due to the low electrochemical activity of the carbon-paste-based electrode composed of a mixture of additives. ${ }^{(17)}$ The porous carbon-particle-modified electrode exhibited a sharper redox peak current and a larger capacitive current than the flat carbon electrode. This means that the porous carbon particles were electrochemically active and the pores had large inner surface areas, which improved the electrochemical properties of the carbon-paste-based flat electrode to increase the electron transfer rate. The significant enhancement of the electrochemical response may be attributed to the large number of edge plane defect sites at the surface of the porous carbon particles that were accessible to the electrolyte. ${ }^{(18)}$ Note that no PVDF binder was included in the porous carbon particle mixture because the peak current decreased as the amount of PVDF mixture increased [Fig. 1(b)]. As confirmed below, the porous carbon particles adhered stably to the surface of the carbon electrode even without PVDF in the measurement solution, possibly owing to the carbon-carbon adhesion. ${ }^{(19)}$ Therefore, PVDF was not used for the GDH/ TB electrodes to avoid the loss of electrochemical activity in the following study.

As described above, the GDH/TB-modified porous carbon electrode was prepared by dropcasting a mixture solution composed of GDH solution and TB solution in a ratio of 1:1 on the porous-carbon-particle-modified electrode. The concentrations of GDH and TB in the mixture solution were optimized to generate the largest response to D-glucose. Figure 2 shows the experimental setup. The GDH/TB-modified porous carbon electrode and $\mathrm{Ag} / \mathrm{AgCl}$ reference electrode were immersed in PBS, and their terminals were short-circuited via an external resistor $(50 \mathrm{k} \Omega)$ to induce redox cycling of TB driven by the difference in the formal redox potentials between these electrodes as demonstrated in our previous report. ${ }^{(14)}$ This configuration produces a reversible and steady-state electric potential difference at the external resistor against a reversible change in D-glucose concentration. In the following section, the change in the electric potential difference at the external resistor represents the sensor response. We adopted this configuration in this study because we plan to combine the present biosensor electrode with a printed organic transistor as an extended-gate electrode in a future study. ${ }^{(14)}$ 


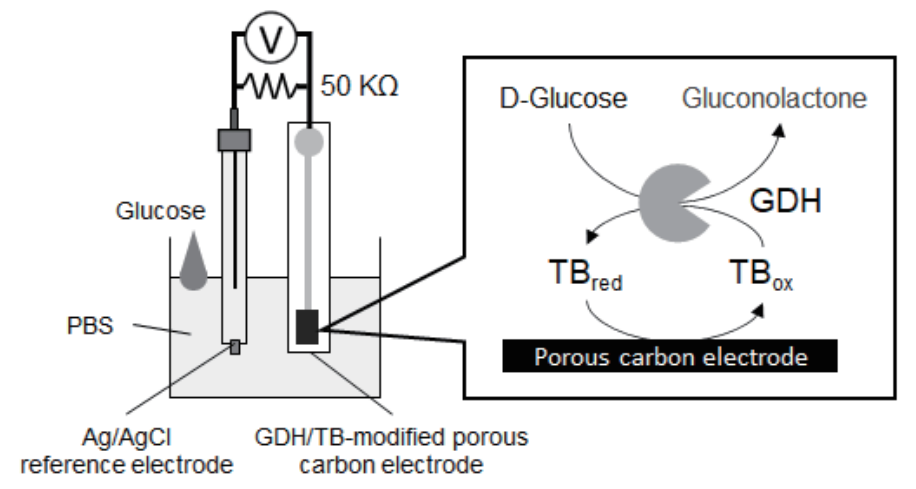

Fig. 2. Setup of the electrochemical measurement using the GDH/TB-modified porous carbon electrode, and the enzymatic reaction generated on the electrode.

Figures 3(a) and 3(b) show the sensor response of the GDH/TB-modified porous carbon electrode to the addition of $10 \mu \mathrm{M}$ D-glucose to the measurement solution for different GDH activities (a) and TB concentrations (b) in the mixture solution. Note that the $y$-axis in Figs. 3(a), 3(b), and 3(d) represents the change in electric potential difference $\Delta V$ at the external resistor because the absolute values of the potential difference were different between the electrodes. However, $\Delta V$ of the separately prepared sensor electrodes changed reproducibly upon the addition of D-glucose as follows. For the data presented in Fig. 3(a), the concentration of TB was set at $10 \mathrm{mM}$. In Fig. 3(b), 50 units $\mu \mathrm{L}^{-1} \mathrm{GDH}$ was used. The sensor response increased with increasing GDH concentration up to 25 units $\mu \mathrm{L}^{-1}$, then saturated above 25 units $\mu \mathrm{L}^{-1}$. In the ensuing experiments, the concentration of GDH was set to 50 units $\mu \mathrm{L}^{-1}$, which is the saturation concentration in the mixture solution. As shown in Fig. 3(b), the sensor response increased up to $5 \mathrm{mM}$ TB. Figure 3(c) shows the CVs acquired in PBS, detected by the GDH/TB-modified porous carbon electrode, with increasing concentration of TB in the mixture solution. The redox peak current increased with increasing concentration of $\mathrm{TB}$, while the peak separation increased, possibly because of the decreased rate of electron self-exchange between TB molecules in the TB multilayer or the reduced diffusion of protons into the TB layer that was used for the redox reaction of TB. On the other hand, we assume that the present biosensor will be used for detecting biomarkers in externally secreted body fluids in the future, where the concentrations change relatively slowly depending on the physiological conditions. Therefore, the TB concentration was determined to be $5 \mathrm{mM}$ to ensure a large sensor response while sacrificing the electron transfer rate of TB. Then, the influence of the immobilization process of FAD-GDH and TB on the sensor response was evaluated as shown in Fig. 3(d). The processes included successive drop-casting from TB to FAD-GDH or from FAD-GDH to TB, and one-step dropcasting of the FAD-GDH/TB mixture on the porous-carbon-modified electrode. The sensor responses were similar for these processes. Therefore, the simple one-step drop-casting of the FAD-GDH/TB mixture on a porous-carbon-modified electrode was selected in the ensuing experiments. 


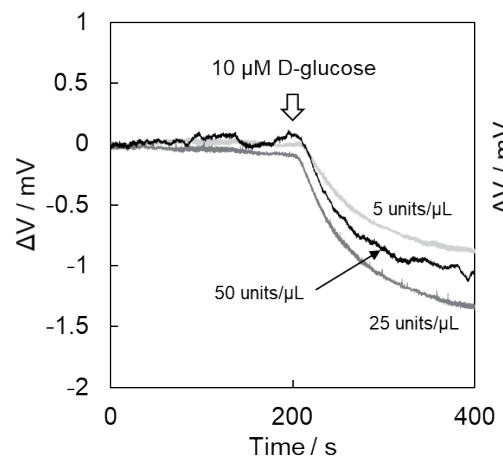

(a)

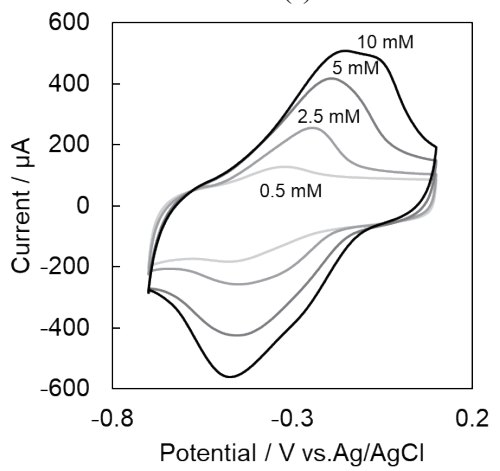

(c)

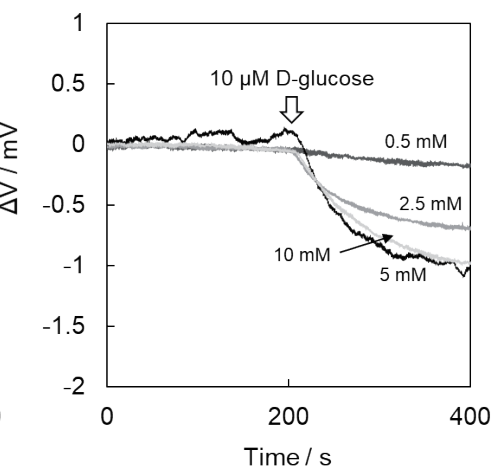

(b)

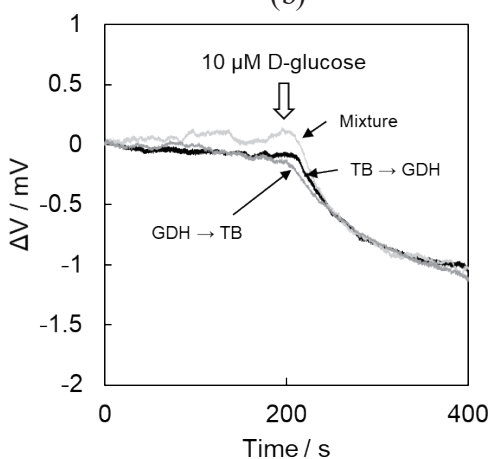

(d)

Fig. 3. Sensor response of the GDH/TB-modified porous carbon electrode to the addition of $10 \mu \mathrm{M}$ D-glucose for different FAD-GDH activities (a) and TB concentrations (b) in the mixture solution. (c) CVs of the GDH/TBmodified porous carbon electrode for different concentrations of TB in the mixture solution. (d) Sensor response of GDH/TB-modified porous carbon electrodes fabricated by different processes.

The immobilization stability of TB and FAD-GDH in the pores of the porous carbon electrode was evaluated over the long term. CV measurements were repeated after 0, 1, 2, 3, 4, 5, and $24 \mathrm{~h}$ and 13 and $21 \mathrm{~d}$ after sensor fabrication. Between measurements, the sensors were stored in PBS at $4{ }^{\circ} \mathrm{C}$. Figure 4(a) shows the CVs of the GDH/TB-modified porous carbon electrode detected in PBS just after fabrication and after $21 \mathrm{~d}$. The CVs were similar even after 21 d. Figure 4(b) shows the relative change in the oxidation peak current of the GDH/TBmodified porous carbon electrode, TB-modified porous carbon electrode, and GDH/TBmodified flat carbon electrode. The GDH/TB-modified porous carbon electrode maintained $91.4 \%$ of the original oxidation peak current even after $21 \mathrm{~d}$, whereas the flat electrode maintained $37.4 \%$ of the original oxidation peak current after $21 \mathrm{~d}$. These results suggest the stable immobilization of GDH and TB in the pores of the porous carbon particles. On the other hand, the peak current of the TB-modified porous carbon electrode decreased to $74.3 \%$, suggesting that the large pores of the porous carbon particles (150 $\mathrm{nm}$ in diameter) containing TB were capped with the assembled small GDH particles [6 $\mathrm{nm}$ in diameter $\left.{ }^{(13)}\right]$.

Figure 5(a) shows the sensor response of the GDH/TB-modified porous carbon electrode to the one-shot injection of D-glucose in the concentration range of $5 \mu \mathrm{M}$ to $1 \mathrm{mM}$. The response 


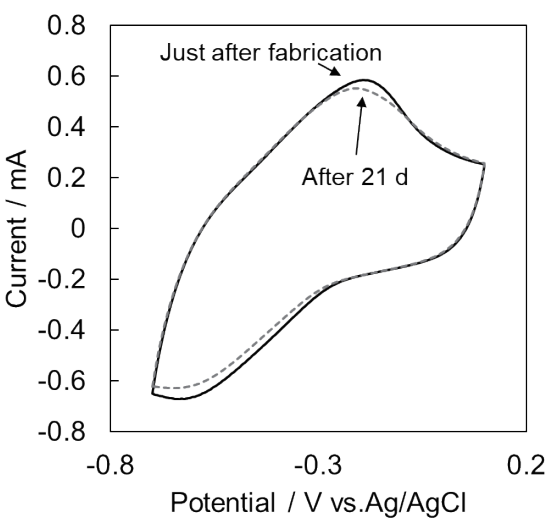

(a)

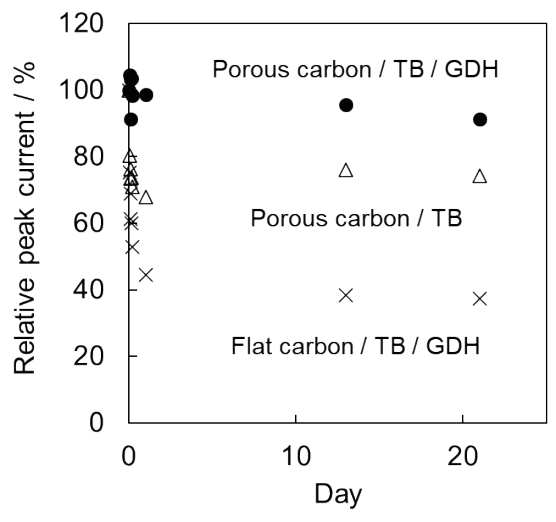

(b)

Fig. 4. (a) CVs of the GDH/TB-modified porous carbon electrode in PBS just after fabrication (solid black line) and after $21 \mathrm{~d}$ (dashed gray line). The scan rate was $0.1 \mathrm{~V} \mathrm{~s}^{-1}$. (b) Time-dependent relative change in oxidation peak current for GDH/TB-modified porous carbon electrode, TB-modified porous carbon electrode, and GDH/TBmodified flat carbon electrode.

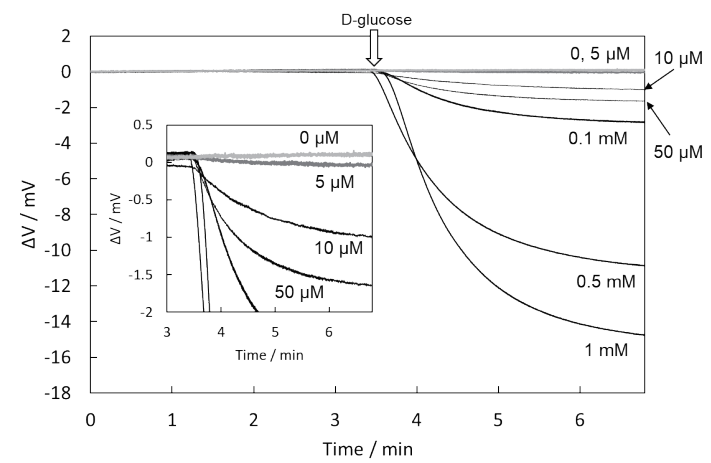

(a)

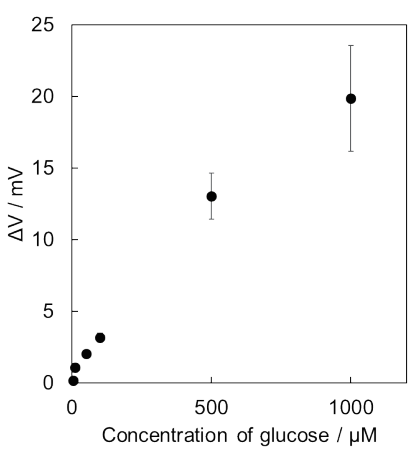

(b)

Fig. 5. (a) Sensor responses of the GDH/TB-modified porous carbon electrode to one-shot injection of D-glucose in the concentration range of $5 \mu \mathrm{M}$ to $1 \mathrm{mM}$. (b) Titration curve for the sensor electrode response versus D-glucose concentration $(n=3)$.

quickly decreased upon the injection of D-glucose and approached a steady state. The $90 \%$ response time for $1 \mathrm{mM}$ D-glucose was $100 \mathrm{~s}$. Figure 5(b) shows the titration curve of the sensor electrode versus the D-glucose concentration. The electrode exhibited a D-glucose concentrationdependent response with good reproducibility $(n=3)$. This result shows that there is little difference in the sensing characteristics of the fabricated GDH/TB-modified porous carbon electrodes. The limit of detection determined from $3 \mathrm{SD} /$ slope in Fig. 5(b) was $6.5 \mu \mathrm{M}$ and the

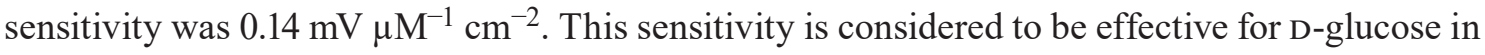
some external bodily fluids such as interstitial fluid $(1.99-22.2 \mathrm{mM})$, urine $(0-0.8 \mathrm{mM})$, and tears $(0.05-5 \mathrm{mM})$, while more sensitivity will be required for D-glucose in the other fluids such as saliva $(0.008-1.77 \mathrm{mM})$ and sweat $(0.01-0.1 \mathrm{mM})$. 
In addition, we examined the sensor response stability of the GDH-TB electrode. The sensor response to one-shot injection of $0.1 \mathrm{mM}$ D-glucose was repeatedly detected for several days. Between measurements, the sensor was stored in PBS at $4{ }^{\circ} \mathrm{C}$. The sensor response (Relative $\Delta V$ for $0.1 \mathrm{mM}$ D-glucose $/ \%$ ) decreased to $38 \%$ two days after the fabrication of the sensor electrode. On the other hand, the relative change in the oxidation peak current for TB at the same time was $115 \%$, that was confirmed in PBS without D-glucose by the same procedure shown in Fig. 4. This means that only GDH activity decreased without the leak of TB from the pores of porous carbon particles. These results suggested that the sensitivity and response stability of the present GDH-TB electrodes are considered to be still insufficient for reliable and long-term monitoring of low concentrations of glucose in externally secreted bodily fluids. We have to improve these characteristics in a future study.

\section{Conclusions}

A sensitive and stable porous-carbon-based enzyme sensor was developed. TB was densely loaded in the pores of porous carbon particles, which were much larger than FAD-GDH particles, and the loaded pores could be stably capped by the enzyme for $21 \mathrm{~d}$. The resulting sensor afforded the reproducible and sensitive detection of D-glucose with a detection limit of $6.5 \mu \mathrm{M}$. However, the present sensitivity and response stability are still insufficient for the reliable monitoring of low concentrations of D-glucose in some externally secreted bodily fluids. In particular, the long-term stability of the enzymatic activity is the issue that needs to be resolved. Some studies have demonstrated the long-term stability of the activity of enzymes loaded into mesoporous carbon, ${ }^{(20)}$ while others examined the modification of the enzyme and its surrounding environment. ${ }^{(21)}$ If these issues are resolved, the present sensor will be promising for the non-invasive and long-term monitoring of low concentrations of biomarkers in externally secreted bodily fluids.

\section{Acknowledgments}

This work was partly supported by the Center of Innovation Program (COI) from the Japan Science and Technology Agency (JST), and by the Foundation of the YU-COE(C) program of Yamagata University.

\section{References}

1 R. Moreddu, D. Vigolo, and A. K. Yetisen: Adv. Healthc Mater. 8 (2019) e1900368. https://doi.org/10.1002/ adhm.201900368

2 T. Gug, M. Tertis, O. Hosu, and C. Cristea: TrAC Trends Anal. Chem. 113 (2019) 301. https://doi.org/10.1016/j. trac.2019.02.020

3 M. Z. Bidin, A. M. Shah, J. Stanslas, and C. L. T. Seong: Clin. Chim. Acta 495 (2019) 239. https://doi. org/10.1016/j.cca.2019.04.069

4 L. B. Baker: Temperature (Austin) 6 (2019) 211. https://doi.org/10.1080/23328940.2019.1632145

5 H. Lee, Y. J. Hong, S. Baik, T. Hyeon, and D. H. Kim: Adv. Healthc. Mater. 7 (2018) e1701150. https://doi. org/10.1002/adhm.201701150

6 E. W. Witkowska, Nery, M. Kundys, P. S. Jeleń, and M. Jönsson-Niedziółka: Anal. Chem. 88 (2016) 11271. https://doi.org/10.1021/acs.analchem.6b03151 
7 J. Tu, R. M. Torrente-Rodríguez, M. Wang, and W. Gao: Adv. Funct. Mater. 30 (2020) 1906713. https://doi. org/10.1002/adfm.201906713

8 M. Etienne, L. Zhang, N. Vilà, and A. Walcarius: Electroanalysis 27 (2015) 2028. https://doi.org/10.1002/ elan.201500172

9 A. Trifonov, K. Herkendell, R. Tel-Vered, O. Yehezkeli, M. Woerner, and I. Willner: ACS Nano 7 (2013) 1358. https://oi.org/10.1021/nn405218x

10 Trifonov, R. Tel-Vered, M. Fadeev, and I. Willner: Adv. Energy Mater. 5 (2015) 1401853. https://doi.org/10.1002/ aenm. 201401853

11 Shitanda, T. Kato, R. Suzuki, T. Aikawa, Y. Hoshi, M. Itagaki, and S. Tsujimura: Bull. Chem. Soc. Jpn. 93 (2020) 32. https://doi.org/10.1246/bcsj.20190212

12 S. Ferri, K. Kojima, and K. Sode: J. Diabetes Sci. Technol. 5 (2011) 1068. https://doi. org $/ 10.1177 / 193229681100500507$

13 H. Lee, Y. S. Lee, S. S. Reginald, S. Baek, E. M. Lee, I. G. Choi, and I. S. Chang: Biosens. Bioelectron. 165 (2020) 112427. https://doi.org/10.1016/j.bios.2020.112427

14 T. Mano, K. Nagamine, Y. Ichimura, R. Shiwaku, H. Furusawa, H. Matsui, D. Kumaki, and S. Tokito: ChemElectroChem 5 (2018) 3881. https://doi.org/10.1002/celc.201801129

15 C. Sandford, M. A. Edwards, K. J. Klunder, D. P. Hickey, M. Li, K. Barman, M. S. Sigman, H. S. White, and S. D. Minteer: Chem. Sci. 10 (2019) 6404. https://doi.org/10.1039/C9SC01545K

16 K. Nagamine, T. Kaya, T. Yasukawa, H. Shiku, and T. Matsue: Sens. Actuators, B 108 (2005) 676. https://doi. org/10.1016/j.snb.2004.10.050

17 G. Cui, J. H. Yoo, J. S. Lee, J. Yoo, J. H. Uhm, G. S. Cha, and H. Nam: Analyst 126 (2001) 1399. https://doi. org/10.1039/B102934G

18 N. Jia, Z. Wang, G. Yang, H. Shen, and L. Zhu: Electrochem. Commun. 9 (2007) 233. https://doi.org/10.1016/j. elecom.2006.08.050

19 Mazurenko, R. Clément, D. Byrne-Kodjabachian, A. de Poulpiquet, S. Tsujimura, and E. Lojou: J. Electroanal. Chem. 8 (2018) 221. https://doi.org/10.1016/j.jelechem.2017.12.041

20 I. Shitanda and S. Tsujimura: J. Phys. Energy 3 (2021) 032002. https://doi.org/10.1088/2515-7655/abebcb

21 S. Rubenwolf, S. Kerzenmacher, R. Zengerle, and F. von Stetten: Appl. Microbiol. Biotechnol. 89 (2011) 1315. https://doi.org/10.1007/s00253-010-3073-6 
